\title{
BOUNDED ANALYTIC FUNCTIONS
}

\section{ZEEV NEHARI}

If $D$ is a domain in the complex $z$-plane, then the family $B=B(D)$ of bounded analytic functions in $D$ is defined as consisting of those analytic functions $f(z)$ which are regular and single-valued in $D$ and which satisfy the inequality $|f(z)|<1$ at all points of $D$. The classical investigations of the family $B(D)$ were restricted to the case in which $D$ is a simply-connected domain. In fact, $D$ was generally taken to be the interior of the unit circle, a restriction which is apparent rather than real since most properties of bounded functions are either invariant with respect to a conformal mapping of $D$, or else are transformed in a simple manner. The use of the simple properties of the unit circle led to a large number of results which are distinguished both by their elegance and their preciseness. However, since the proofs leading to these results lean heavily on the special features of the unit circle, they gave little or no indication as to their possible generalization to the case of bounded functions in multiply-connected domains.

In the classical treatment of bounded functions, the family $B$ was occasionally replaced by the more general class of analytic functions $w=f(z)$ whose values-for $z \in D$-are contained in a specified simplyconnected domain $D^{\prime}$ in the $w$-plane. The family $B$ corresponds to the case in which $D^{\prime}$ is the unit circle $|w|<1$. Other special cases are the family of functions with a positive real part-to be denoted by $P=P(D)$-obtained if $D^{\prime}$ is the right half-plane $\operatorname{Re}\{w\}>0$, and the family of functions with a bounded real part-denoted by $B_{R}=B_{R}(D)$-for which $D^{\prime}$ is the infinite strip $-1<\operatorname{Re}\{w\}<1$. These families are obtained from $B$ by means of the conformal transformations which carry $|w|<1$ into the various domains $D^{\prime}$. For instance, we have

$$
\begin{aligned}
g(z)=\frac{1+f(z)}{1-f(z)}, & f(z) \in B, g(z) \in P, \\
\phi(z)=\frac{4}{\pi} \arctan f(z), & f(z) \in B, \phi(z) \in B_{R} .
\end{aligned}
$$

Apart from their intrinsic interest, these classes are often useful in the investigation of the functions of $B$ since the special features of

An address delivered before the Cincinnati meeting of the Society, February 23, 1951, by invitation of the Committee to Select Hour Speakers for Western Sectional Meetings; received by the editors June 2, 1951. 
the classes $P$ and $B_{R}$ make it possible to use certain simple yet powerful techniques whose adaptation to the class $B$ would be a matter of considerable difficulty.

The novel features encountered in the attempt to generalize the classical results on bounded functions to the case of multiply-connected domains are due to the failure of the monodromy theorem. We are therefore faced by two different types of problems, according as we consider the family of all functions which are regular and bounded in $D$, or confine ourselves to those functions which are, in addition, single-valued in $D$. The first case introduces no new features since, by considering the function on the universal covering surface of $D$, we may reduce it to the simply-connected case. In the case of single-valued functions, however, this device is obviously unable to yield precise results, and different methods have to be used.

The first to consider problems of this kind seem to have been Carlson [5 $]^{1}$ and Teichmüller [20] who obtained a sharpened version of Hadamard's three circle theorem for functions which are singlevalued in a circular ring, and Heins [11] who considered a number of extremal problems for functions regular and single-valued in a doubly-connected domain. The case of bounded functions in domains of arbitrary finite connectivity was first treated by Grunsky $[9,10]$, who considered generalizations of the lemmas of Schwarz, Julia, and Loewner. The extension of Schwarz' lemma aimed at by Grunsky consists in the following problem: Given a domain $D$ of connectivity $n$ and two distinct points - say $\zeta$ and $z_{0}$ - of $D$, to find a function $f_{0}(z)$ of $B(D)$ such that $f_{0}(\zeta)=0$ and $\left|f\left(z_{0}\right)\right| \leqq\left|f_{0}\left(z_{0}\right)\right|$, where $f(z)$ is any other function of $B(D)$ which vanishes at $\zeta$. The existence of such a function $f_{0}(z)$ follows, of course, from the compactness of the family $B(D)$. Grunsky showed [10] that the extremal function $w=f_{0}(z)$ yields a conformal mapping of $D$ onto the $n$-times covered unit circle $|w|<1$.

The existence of such mappings had been conjectured by Riemann and it was proved later $[4,8]$ that there exist a large variety of essentially different conformal mappings of a domain of connectivity $n$ onto the $m$-times covered unit circle, where $m \geqq n$. In [10] it is shown that any function of $B$ can be approximated by functions mapping $D$ onto the multiply-covered unit circle and that it is therefore sufficient to consider functions of this latter type. The solution of the problem $\left|f\left(z_{0}\right)\right|=\max , f(\zeta)=0$ among these functions is then shown to have not more than $n-1$ zeros in $D$ other than $\zeta$, and this

\footnotetext{
${ }^{1}$ Numbers in brackets refer to the references cited at the end of the paper.
} 
is equivalent to the characterization of the function $f_{0}(z)$ given above.

The fact that the extremal function yields a $(1, n)$ mapping of $D$ onto the unit circle is not sufficient to characterize the extremal function completely, since there exist an infinity of essentially different mappings of this type. The problem thus remains of characterizing the extremal mapping within this class. This problem was solved by Ahlfors [1], who considered a generalization of the Schwarz lemma slightly different from the one mentioned above, namely, the problem: $f(z) \in B(D), \zeta \in D,\left|f^{\prime}(\zeta)\right|=\max$. Ahlfors shows that the extremal function $F(z)$ yields a $(1, n)$ mapping of $D$ onto the unit circle and that the zeros $\zeta, z_{1}, z_{2}, \cdots, z_{n-1}$ of $F(z)$ in $D$ are distinguished by the fact that there exists a differential $p^{\prime}(z) d z$ such that

$$
\frac{1}{i} p^{\prime}(z) d z>0,
$$$$
z \in C
$$

( $C$ being the boundary of $D$ ), where $p^{\prime}(z)$ is regular in $D$ except for a simple pole of residue 1 at $\zeta$ and $z_{1}, \cdots, z_{n-1}$ are the $n-1$ zeros of $p^{\prime}(z)$ in $D$ (the fact that a function $p^{\prime}(z)$ with one pole in $D$ and with the boundary behavior (3) has precisely $n-1$ zeros in $D$ is an immediate consequence of the argument principle). We remark that in the case in which the boundary $C$ of $D$ consists of $n$ closed analytic curves-and it is sufficient to consider this case-it follows from (3) and the Schwarz reflection principle that $p^{\prime}(z)$ is regular on C.

The proof of this property of the extremal is based on the observation that the function $\log |F(z)|$ is-apart from a number of logarithmic poles - a harmonic function in $D$ whose harmonic conjugate has periods about the boundary components of $D$ which are integral multiples of $2 \pi$. By slight variations of the locations of the logarithmic poles-which amounts to adding and subtracting Green's functions of $D$ with suitable points of reference-and by variations of the boundary values of $\log |F(z)|$, a function $\log \left|F^{*}(z)\right|$ is obtained. In order to insure that $F^{*}(z)$ is also single-valued in $D$ and is thus a competing function in the original problem, it is necessary to carry the condition that the periods of the harmonic conjugate of $\log |F(z)|$ remain unchanged in these variations. This is achieved by a Lagrange multiplier technique, which finally leads to the above result. In a more recent paper [2], Ahlfors shows that a similar procedure can be applied to bounded functions on an open Riemann surface of genus higher than zero, and that it leads to similar results. The extremal map is again a covering surface of the unit circle; if $p$ is the genus 
of the surface and $n$ is the number of its boundary components, then the number $m$ of the sheets of the extremal map satisfies $n \leqq m \leqq n$ $+2 p$.

The investigation of the extremal problem $f(z) \in B(D),\left|f^{\prime}(\zeta)\right|$ $=$ max was carried further by Garabedian [6] who showed that there exists an interesting connection between this problem and the problem

$$
\int_{C}|h(z)| d s=\min
$$

where $h(z)$ is regular in $D$ except for a double pole at $\zeta$ with the principal part $(z-\zeta)^{-2}$, and where the boundary behavior of $h(z)$ is such that the integral in (4) exists. To see this, consider the function $q(z)$ defined by

$$
q(z)=\frac{p^{\prime}(z) F^{\prime}(\zeta)}{F(z)}
$$

where $F(z)$ is Ahlfors' extremal function and $p^{\prime}(z)$ is the function appearing in (3). Since the $n-1$ zeros of $p^{\prime}(z)$ coincide with the zeros of $F(z)$ other than $z=\zeta, q(z)$ has no zeros and is regular in $D$ except for a double pole at $z=\zeta$. It follows from (3) and (5) that the boundary relation

$$
-i F(z) q(z) d z>0
$$$$
z \in C
$$

holds. The principal part of $q(z)$ at $z=\zeta$ is $(z-\zeta)^{-2}$. Indeed, if there were a term of the form $\alpha(z-\zeta)^{-1}$, it would follow from (6) and the fact that $|F(z)|=1$ on $C$ that $^{2}$

$$
\begin{aligned}
2 \pi \alpha & =\frac{1}{i} \int_{C} q(z) d z=\frac{1}{i} \int_{C}[F(z)]^{*} F(z) q(z) d z \\
& =\frac{1}{i}\left[\int_{C} F^{2}(z) q(z) d z\right]^{*},
\end{aligned}
$$

and this vanishes since, in view of $F(\zeta)=0$, the integrand is regular in $D$.

The fact that $F(z)$ is the extremal function of the generalized Schwarz lemma and that $q(z)$ solves the problem (4) was deduced by Garabedian from (6) in the following elegant manner. If $f(z)$ is a function of $B(D)$, it follows from the residue theorem and the inequality (6) that

${ }^{2}$ Complex conjugates are denoted by asterisks. 


$$
\begin{aligned}
\left|f^{\prime}(\zeta)\right| & =\left|\frac{1}{2 \pi i} \int_{C} f(z) q(z) d z\right| \leqq \frac{1}{2 \pi} \int_{C}|q(z) d z| \\
& =\frac{1}{2 \pi} \int_{C}\left|\frac{1}{i} F(z) q(z) d z\right|=\frac{1}{2 \pi i} \int_{C} F(z) q(z) d z=F^{\prime}(\zeta)
\end{aligned}
$$

where the fact that $|F(z)|=1, z \in C$, has been used. This shows the extremal property of $F(z)$. As to the problem (4), it follows from the properties of the functions $h(z)$ that

$$
\int_{C}|h(z)| d s \geqq\left|\int_{C} h(z) F(z) d z\right|=2 \pi F^{\prime}(\zeta) .
$$

By (7), we have

$$
\int_{C}|q(z)| d s=2 \pi F^{\prime}(\zeta)
$$

and therefore

$$
\int_{C}|q(z)| d s \leqq \int_{C}|h(z)| d s .
$$

Thus, $q(z)$ solves the extremal problem (4), and the extremal values of (4) and of the quantity associated with the generalization of Schwarz' lemma are connected by the simple relation (8).

The fact that our maximum problem in the theory of bounded functions is associated with a "dual" minimum problem of the type (4) is not an isolated occurrence. This duality was recognized by Fr. Riesz [18] in a variety of extremal problems concerned with bounded functions in the unit circle. In all these cases, the two associated extremal functions form a positive differential in the manner of (6), and their extremal properties follow from the positivity of the differential by judicious use of the residue theorem. In the case of the unit circle, the existence of pairs of functions with suitable properties which form positive differentials is trivial and they can easily be written down explicitly in terms of elementary functions. In the case of general multiply-connected domains $D$ this is not true any more and the relevant existence theorems appear to lie somewhat deeper than the existence theorems for the usual harmonic and analytic domain functions of $D$. A direct, but rather difficult, proof for the existence of two functions $F(z)$ and $q(z)$ which are connected by (6) and have the other properties specified above was given by Garabedian and Schiffer [7]. A short proof which, however, uses the existence of the Green's function of $D$ can be found in [15]. 
Another important aspect of the problem $f(z) \in B(D),\left|f^{\prime}(\zeta)\right|$ $=\max$ is the connection between the extremal $F(z)$ and the Szegö kernel function [19] established in [6]. It is not difficult to show that the function $q(z)$ in (6) can be written in the form $q(z)=4 \pi^{2} L^{2}(z, \zeta)$, where $L(z, \zeta)$ is regular and single-valued in $D+C$ except for a simple pole of residue $(2 \pi)^{-1}$ at $z=\zeta$. If we denote the regular function $F(z) L(z, \zeta)^{\prime}$ by $K(z, \zeta)$, that is, we write

$$
F(z)=\frac{K(z, \zeta)}{L(z, \zeta)}
$$

we can deduce from $(6)$ that $K(z, \zeta)$ and $L(z, \zeta)$ are connected by the relation

$$
[K(z, \zeta)]^{*} d s=-i L(z, \zeta) d z, \quad z \in C .
$$

If $\phi(z)$ is any function which is regular and single-valued in $D$ and such that $|\phi(z)|^{2}$ can be integrated over $C$, it follows from (10) and the residue theorem that

$$
\int_{C}[K(z, \zeta)]^{*} \phi(z) d s=\frac{1}{i} \int_{C} L(z, \zeta) \phi(z) d z=\phi(\zeta) .
$$

The function $K(z, \zeta)$ has therefore the characteristic reproducing property

$$
\phi(\zeta)=\int_{C}[K(z, \zeta)]^{*} \phi(z) d s
$$

of the Szegö kernel function [19] of $D$ and is thus identical with it. Since, by (9),

$$
F^{\prime}(\zeta)=2 \pi K(\zeta, \zeta)
$$

and since kernel functions can be numerically computed in terms of complete orthonormal sets of functions [3], (11) yields the complete numerical solution of the problem $f(z) \in B(D),\left|f^{\prime}(\zeta)\right|=\max$.

As pointed out above, it is useful to consider also functions of the classes $P$ and $B_{R}$, which are related to the functions of $B$ by the equations (1) and (2), respectively. The special properties of these classes make it possible to devise methods for the solution of extremal problems which are considerably simpler than those required for a direct attack on the class $B[14,17]$. To illustrate the method appropriate for problems within the class $P$ [14], we consider the extremal problem $g(z) \in P, g(\zeta)=1,\left|g^{\prime}(\zeta)\right|=\max$ which is identical with the generalization of Schwarz' lemma discussed further above. From a 
trivial transformation it follows that it is sufficient to consider the problem $g^{\prime}(\zeta)=$ max within the subclass of functions of $P$ for which $g^{\prime}(\zeta)>0$. Let now $-i r^{\prime}(z) d z$ be a non-negative differential on $C$, that is,

$$
-i r^{\prime}(z) d z \geqq 0, \quad z \in C,
$$

where $r^{\prime}(z)$ is regular and single-valued in $D$ (and, by the reflection principle, also on $C$ ) except at $z=\zeta$ where

$$
r^{\prime}(z)=-\frac{1}{(z-\zeta)^{2}}+\frac{\alpha}{z-\zeta}+r_{1}(z)
$$

and $r_{1}(z)$ is regular. In view of (12), $\alpha$ is necessarily positive. If $g(z)$ is a function of $P$ which is integrable over $C$, it follows from (12), (13), and the residue theorem that

$$
\begin{aligned}
\operatorname{Re}\left\{\alpha-g^{\prime}(\zeta)\right\} & =\operatorname{Re}\left\{\frac{1}{2 \pi i} \int_{C} g(z) r(z) d z\right\} \\
& =\frac{1}{2 \pi i} \int_{C} \operatorname{Re}\{g(z)\} r(z) d z \geqq 0,
\end{aligned}
$$

where the fact that $\operatorname{Re}\{g(z)\} \geqq 0$ has been used. Since both $\alpha$ and $g^{\prime}(\zeta)$ are positive we find that

$$
g^{\prime}(\zeta) \leqq \alpha .
$$

In order to obtain the best estimate of this type, we have to use the function (13) for which $\alpha=\min$ under the condition (12). This minimum problem presents no difficulties since the positive differentials (12) form an $n$-parameter family which can be expressed in terms of the Green's function and the harmonic measures of $D$. It is found that the minimizing differential is characterized by the fact that the associated function $r^{\prime}(z)$ has a double zero-say $z_{\nu}$-on each boundary component $C_{\nu}(\nu=1, \cdots, n)$ of $D$. For the $\alpha$ belonging to this differential, the inequality (14) will then be sharp, and the extremal $w=G(z)$ of our original problem will necessarily yield a $(1, n)$ mapping of $D$ onto the right half-plane $\operatorname{Re}\{w\}>0$. Indeed, as shown in $[4,8]$, there exists a completely determined mapping $w=f(z)$ of $D$ onto the $n$-times covered unit circle such that $f(\zeta)=0, f^{\prime}(\zeta)>0$, and $f\left(z_{\nu}\right)=1, \nu=1, \cdots, n$, where $z_{\nu} \in C_{\nu}$. Passing to the class $P$ by means of (1), we obtain a function $w=G(z)$ which yields a $(1, n)$ mapping of $D$ onto $\operatorname{Re}\{w\}>0$ and whose only singularities on $C$ are simple poles at the points $z_{\nu}$. Since these are the zeros of the minimizing 
differential $r^{\prime}(z)$, we may evaluate the integral

$$
\frac{1}{2 \pi i} \int_{C} G(z) r^{\prime}(z) d z
$$

by the residue theorem. In view of (12), (13), and the fact that $\operatorname{Re}\{G(z)\}=0$ for $z \in C, z \neq z_{\nu}$, we obtain $\alpha-G^{\prime}(\zeta)=0$, which shows that (14) is sharp and that $G(z)$ solves our problem.

By using positive differentials which show a singular behavior different from that indicated in (13), it is possible to solve a large variety of extremal problems within the family $P$. In all these cases, the extremal functions yield mappings of $D$ onto covering surfaces of the right half-plane [14]. It is interesting to note that while the extremal function of the Schwarz lemma is essentially unique, there will be no uniqueness in the case of those problems whose associated positive differentials have more than two poles in $D$.

In the case of the class $B_{R}$, that is, the class of the functions $\phi(z)$ which are regular and single-valued in $D$ and satisfy $|\operatorname{Re}\{\phi(z)\}|<1$, different methods are indicated [17]. If we write $\phi(z)=u(z)+i v(z)$, where $u(z)$ and $v(z)$ are real, we are dealing with those harmonic functions $u(z)$ which satisfy $|u(z)|<1$ in $D$ and which possess a singlevalued harmonic conjugate. The latter condition is equivalent to the $n-1$ conditions

$$
\int_{C} u(z) \frac{\partial \omega_{\nu}(z)}{\partial n} d s=0, \quad \nu=1, \cdots, n-1,
$$

where $\omega_{\nu}(z)$ is the harmonic measure associated with $C_{\nu}$, that is, $\omega_{\nu}(z)$ is harmonic in $D$ and has the boundary values $\delta_{\nu \mu}$ on $C_{\mu}$. To illustrate the method of [17] by a simple example, we consider the problem $\phi(\zeta)=0,|\phi(\eta)|=\max (\zeta, \eta \in D, \zeta \neq \eta)$ which is equivalent to the generalization of Schwarz' lemma treated in [10]. From trivial transformations it follows that it is sufficient to consider the problem $u(\zeta)$ $=0, u(\eta)=\max$. Using Green's formula and taking into account the conditions (15), we have

$$
u(\eta)=-\frac{1}{2 \pi} \int_{C} u(z)\left[\frac{\partial g(z, \eta)}{\partial n}+\alpha \frac{\partial g(z, \zeta)}{\partial n}\right.
$$

$$
\left.+\sum_{\nu=1}^{n-1} \lambda_{\nu} \frac{\partial \omega_{\nu}(z)}{\partial n}\right] d s
$$

where $g(z, \eta)$ is the Green's function of $D$ and $\alpha$ and the $\lambda_{\nu}$ are arbitrary real parameters. Since $|u(z)| \leqq 1$, it follows from (15) that 


$$
\begin{aligned}
u(\eta) & \leqq \frac{1}{2 \pi} \int_{C}\left|\frac{\partial g(z, \eta)}{\partial n}+\alpha \frac{\partial g(z, \zeta)}{\partial n}+\sum_{\nu=1}^{n-1} \lambda_{\nu} \frac{\partial \omega_{\nu}(z)}{\partial n}\right| d s \\
& =\frac{1}{2 \pi} \int_{C}|P| d s
\end{aligned}
$$

where $P$ is an abbreviation for

$$
P=\frac{\partial g(z, \eta)}{\partial n}+\alpha \frac{\partial g(z, \zeta)}{\partial n}+\sum_{\nu=1}^{n-1} \lambda_{\nu} \frac{\partial \omega_{\nu}(z)}{\partial n}, \quad z \in C .
$$

To obtain the best possible inequality (17), we minimize the righthand side of (17) with respect to the arbitrary parameters $\alpha, \lambda_{1}, \cdots$, $\lambda_{n-1}$. This leads to the conditions

$$
\begin{aligned}
& \int_{C} \operatorname{sgn} P \frac{\partial g(z, \zeta)}{\partial n} d s=0, \\
& \int_{C} \operatorname{sgn} P \frac{\partial \omega_{\nu}(z)}{\partial n} d s=0, \quad \nu=1, \cdots, n-1,
\end{aligned}
$$

where $\operatorname{sgn} P$ is equal to 1 or -1 , according as $P$ is positive or negative, and remains undetermined if $P=0$. If we introduce a harmonic function $V(z)$ by the Dirichlet problem

(19) $U(z)=-\operatorname{sgn} P, \quad z \in C, P \neq 0, \quad|U(z)| \leqq 1, \quad z \in D$,

these conditions read

$$
\begin{aligned}
& \int_{C} U(z) \frac{\partial g(z, \zeta)}{\partial n} d s=0 \\
& \int_{C} U(z) \frac{\partial \omega_{\nu}(z)}{\partial n} d s=0, \quad \nu=1, \cdots, n-1
\end{aligned}
$$

(20) shows that $U(\zeta)=0$, while a comparison of (21) and (15) shows that the harmonic conjugate $V(z)$ of $U(z)$ is single-valued in $D$. The analytic function $\phi(z)=U(z)+i V(z)$ thus belongs to $B_{R}$. From (19) and the easily demonstrable fact that an expression of the type (18) cannot have more than $2 n$ zeros on $C$, it further follows that $w=\phi(z)$ yields a $(1, n)$ mapping of $D$ onto the infinite strip -1 $<\operatorname{Re}\{w\}<1$. The function $\phi(z)$ solves our extremal problem. Indeed, we have $U(\zeta)=0$ and, in view of (17), (19), and (18),

$$
u(\eta) \leqq \frac{1}{2 \pi} \int_{C}|P| d s=-\frac{1}{2 \pi} \int_{C} U(z) P d s=U(\eta) .
$$


This method is easily extended to the case in which the class $B_{R}$ is restricted by various types of side conditions. As an example, we mention the problem: $\phi(z) \in B_{R}, \phi\left(a_{\mu}\right)=b_{\mu}, \mu=1, \cdots, m,|\phi(\zeta)|$ $=\max$ where we have to require, of course, that there exist at least one function of $B_{R}$ which satisfies the given interpolation conditions. The extremal is found to yield a $(1, k)$ mapping of $D$ onto the strip $-1<\operatorname{Re}\{w\}<1$, where $n \leqq k \leqq n+m-1$. This result, which represents a generalization of the well known Pick-Nevanlinna interpolation theory, was first proved-by different methods-by Garabedian [6]. Another derivation, based on a lemma on positive harmonic functions, has recently been given by Heins [12].

By suitable modifications of the basic method of proof in [17], a large number of extremal problems in the class $B_{R}$ and in related classes can be treated. For instance, if $\phi(z) \in B_{R}, \phi^{\prime}(\zeta)=A$ is given and $\eta$ is such that $\phi^{\prime}(\eta)$ cannot vanish, then the problem $\left|\phi^{\prime}(\eta)\right|=\min$ is solved by a function mapping $D$ onto the $m$-times covered strip $-1<\operatorname{Re}\{w\}<1$, where $n \leqq m \leqq n+2$. A similar result is obtained if the condition $\left|\phi^{\prime}(\eta)\right|=\min$ is replaced by $|\phi(u)-\phi(v)|=\min$, where the points $u$ and $v$ are such that $\phi(u)$ and $\phi(v)$ cannot coincide if $\phi^{\prime}(\zeta)=A$. From the last two results it is not difficult to deduce a result concerning the radius of univalence about a given point $\zeta$ of those functions $f(z)$ of $B$ for which $\left|f^{\prime}(\zeta)\right| \geqq A$, where $A$ is a given positive number. It is found that, among the functions satisfying these conditions, the smallest radius of univalence is obtained for a function mapping $D$ onto the $m$-times covered unit circle, where $n \leqq m \leqq n+2$. This generalizes a classical result of Landau [13] for the case $n=1$, in which $m=2$.

The class $B$ can be generalized in a number of ways. One generalization is obtained by replacing the condition $|f(z)|<1, z \in D$, by $\lim \sup _{z \rightarrow z_{0}}|f(z)| \leqq \lambda\left(z_{0}\right)$, where $\lambda\left(z_{0}\right)$ is a positive continuous function on each boundary component of $D$ and $z$ approaches the boundary point $z_{0}$ from inside $D$ [16]. The discussion of the problem $\left|f^{\prime}(\zeta)\right|=\max$ in this class of functions leads to two domain functions $K_{\lambda}(z, \zeta)$ and $L_{\lambda}(z, \zeta)$ which generalize the functions $K(z, \zeta)$ and $L(z, \zeta)$ associated with the analogous problem in the class $B . K_{\lambda}(z, \zeta)$ is regular in $D, L_{\lambda}(z, \zeta)$ is regular in $D$ except for a simple pole of residue $(2 \pi)^{-1}$ at $z=\zeta$, and on the boundary we have the identity

$$
\left[K_{\lambda}(z, \zeta)\right]^{*} d s=-i \lambda(z) L(z, \zeta) d z, \quad z \in C,
$$

which generalizes (10). The maximal value of $\left|f^{\prime}(\zeta)\right|$ is given by $2 \pi K_{\lambda}(\zeta, \zeta)$. If $g(z)$ is regular and single-valued in $D$ and $|g(z)|$ is square-integrable on $C$, it follows from (22) and the residue theorem 
that

$$
\int_{C} \lambda^{-1}(z) g(z)\left[K_{\lambda}(z, \zeta)\right]^{*} d s=g(\zeta)
$$

This shows that $K_{\lambda}(z, \zeta)$ is a kernel function which can be computed by the expansion

$$
K_{\lambda}(z, \zeta)=\sum_{\nu=1}^{\infty} g_{\nu}(z)\left[g_{\nu}(\zeta)\right]^{*}
$$

where the $g_{\nu}(z)$ are a complete set of functions with the same propperties as $g(z)$ which are orthonormalized by the conditions

$$
\int_{C} \lambda^{-1}(z) g_{\nu}(z)\left[g_{\nu}(z)\right] * d s=\delta_{\nu \mu}
$$

Another generalization of the class $B$ is obtained by extending $B$ to include, in addition to bounded analytic functions, also bounded complex harmonic functions which are not necessarily analytic. This class, say $B^{\prime}$, will thus consist of functions of the form $f(z)+g^{*}(z)$, where $f(z)$ is analytic and $g^{*}(z)$ is the complex conjugate of an analytic function, such that

$$
\left|f(z)+g^{*}(z)\right|<1, \quad z \in D .
$$

Both $f(z)$ and $g(z)$ are supposed single-valued in $D$.

The treatment of the class $B^{\prime}$ can be modelled on that of the class $B_{R}$. To illustrate the procedure, we consider the problem $\mid f^{\prime}(\zeta)$ $+\left[g^{\prime}(\zeta)\right]^{*} \mid=\max , \zeta \in D$. By Green's formula, we have

$$
\begin{aligned}
f^{\prime}(\zeta)+\left[g^{\prime}(\zeta)\right]^{*} & \\
= & -\frac{1}{2 \pi} \int_{C}\left[f(z)+g^{*}(z)\right]\left[\frac{\partial^{2} g(z, \zeta)}{\partial n \partial \xi}+\sum_{\nu=1}^{n-1} \frac{\partial \omega_{\nu}(z)}{\partial n}\right] d s, \\
\zeta & =\xi+i \eta,
\end{aligned}
$$

where the arbitrary complex constants $\lambda_{\nu}$ take account of the fact that $f(z)$ and $g(z)$ are single-valued in $D$. By (23), it follows that

$$
\left|f^{\prime}(\zeta)+\left[g^{\prime}(\zeta)\right]^{*}\right| \leqq \frac{1}{2 \pi} \int_{C}|P| d s,
$$

where

$$
P=\frac{\partial^{2} g(z, \zeta)}{\partial n \partial \xi}+\sum_{\nu=1}^{n-1} \lambda_{\nu} \frac{\partial \omega_{\nu}(z)}{\partial n}
$$


We now minimize the right-hand side of (25) with respect to the arbitrary complex parameters $\lambda_{\nu}$. This leads to the necessary conditions

$$
\int_{C} \exp \{i \arg P\} \frac{\partial \omega_{\nu}}{\partial n} d s=0, \quad \nu=1, \cdots, n-1 .
$$

If we define a complex harmonic function $h(z)=u(z)+i u_{1}(z)$ by the boundary value problem

$$
h(z)=\exp \{-i \arg P\}, \quad z \in C,
$$

this condition reads

$$
\int_{C} u \frac{\partial \omega_{\nu}}{\partial n} d s+i \int_{C} u_{1} \frac{\partial \omega_{\nu}}{\partial n} d s=0, \quad \nu=1, \cdots, n-1 .
$$

This shows that the harmonic conjugates $v(z)$ and $v_{1}(z)$ of $u(z)$ and $u_{1}(z)$, respectively, are single-valued. Hence, $2 u(z)=p(z)+p^{*}(z)$ and $2 i u_{1}(z)=q(z)-q^{*}(z)$, where the analytic functions $p_{*}(z)$ and $q(z)$ are single-valued in $D$. The function $h(z)$ defined by $(27)$ is therefore of the form

$$
\begin{aligned}
h(z) & =\frac{1}{2}[p(z)+q(z)]+\frac{1}{2}[p(z)-q(z)]^{*} \\
& =F(z)+G^{*}(z),
\end{aligned}
$$

where $F(z)$ and $G(z)$ are regular and single-valued in $D$. Since, by (27), $|h(z)|=1$ on $C$ and since the maximum principle is also valid for complex harmonic functions, it follows thus that $h(z)$ belongs to $B^{\prime}$.

That the function $h(z)$ solves our extremal problem is now easily seen. In view of (27), we have

$$
|P|=P h(z), \quad z \in C .
$$

Inserting this in (25), and observing (24), (26), and (28), we obtain

$$
\left|f^{\prime}(\zeta)+\left[g^{\prime}(\zeta)\right]^{*}\right| \leqq F^{\prime}(\zeta)+\left[G^{\prime}(\zeta)\right]^{*}
$$

which expresses the extremal property. As in the case of the corresponding problem within the class $B$, the extremal function is associated with a positive differential. Since $g(z, \zeta)$ and $\omega_{\nu}(z)$ are constant on each boundary component of $D$, the expression $P$ defined in (26) satisfies

$$
P d s=-i Q(z) d z, \quad z \in C,
$$


where $Q(z)$ is a single-valued analytic function which is regular in $D$ except for a double pole with the meromorphic part $(z-\zeta)^{-2}$. Combining this with (29) and (28), we find

$$
-i\left[F(z)+G^{*}(z)\right] Q(z) d z \geqq 0,
$$

a boundary relation analogous to (6).

\section{REFERENCES}

1. L. V. Ahlfors, Bounded analytic functions, Duke Math. J. vol. 14 (1947) pp. 1-11.

2. - Open Riemann surfaces and extremal problems on compact subregions, Comment. Math. Helv. vol. 24 (1950) pp. 100-134.

3. S. Bergman, The kernel function and conformal mapping, New York, American Mathematical Society, 1950.

4. L. Bieberbach, Ueber einen Riemannschen Satz aus der Lehre von der konformen Abbildung, Berlin Math. Ges. Sitzungsber. vol. 24 (1925) pp. 6-9.

5. F. Carlson, Sur le module maximum d'une fonction analytique uniforme, Arkiv für Mathematik, Astronomi och Fysik vol. 26 (1938) no. 9, pp. 1-13.

6. P. R. Garabedian, Schwarz' lemma and the Szegö kernel function, Trans. Amer. Math. Soc. vol. 67 (1949) pp. 1-35.

7. P. R. Garabedian and M. Schiffer, On existence theorems of potential theory and conformal mapping, Ann. of Math. vol. 52 (1950) pp. 164-187.

8. H. Grunsky, Ueber die konforme Abbildung mehrfach zusammenhängender Gebiete auf mehrblättrige Kreise, Preuss. Akad. Wiss. Sitzungsber. (1937) pp. 40-46.

9. - Eindeutige beschränkte Funktionen in mehrfach zusammenhängenden Gebieten I, Jber. Deutschen Math. Verein. vol. 50 (1940) pp. 230-255.

10. - Eindeutige beschränkte Funktionen in mehrfach zusammenhängenden Gebieten II, Jber. Deutschen Math. Verein. vol. 52 (1942) pp. 118-132.

11. M. Heins, Extremal problems for functions analytic and single-valued in a doublyconnected region, Amer. J. Math. vol. 42 (1940) pp. 91-106.

12. - A lemma on positive harmonic functions, Ann. of Math. vol. 52 (1950) pp. 568-573.

13. E. Landau, Der Picard-Schottkysche Satz und die Blochsche Konstante, Preuss. Akad. Wiss. Sitzungsber. (1926) pp. 467-474.

14. Z. Nehari, Analytic functions possessing a positive real part, Duke Math. J. vol. 15 (1948) pp. 1033-1042.

15. - On bounded analytic functions, Proceedings of the American Mathematical Society vol. 1 (1950) pp. 268-275.

16. - - A class of domain functions and some allied extremal problems, Trans. Amer. Math. Soc. vol. 69 (1950) pp. 161-178.

17. - Extremal problems in the theory of bounded analytic functions, Amer. J. Math. vol. 73 (1951) pp. 78-106.

18. F. Riesz, Ueber Potenzreihen mit vorgeschriebenen Anfangsgliedern, Acta Math. vol. 42 (1920) pp. 145-171.

19. G. Szegö, Ueber orthogonale Polynome, die zu einer gegebenen Kurve der komplexen Ebene gehören, Math. Zeit. vol. 9 (1921) pp. 218-270.

20. O. Teichmüller, Eine Verschärfung des Dreikreisesatzes, Deutsche Mathematik vol. 4 (1939) pp. 16-22.

WASHINGTON UNIVERSITY 